Pak. j. sci. ind. res. Ser. B: biol. sci. 201356 (1) 53-55

\title{
Study of Human Cystic Echinococcosis in Hyderabad, Pakistan
}

\author{
Lochi Ghulam Murtaza ${ }^{a}$, Muhammad Ghiasuddin Shah ${ }^{\mathrm{a}}$, Amjad Hussain Merania, \\ Muhammad Shoaib Khan ${ }^{b *}$, Sheeraz Mustafa Khushk ${ }^{a}$ and Abdul Manan Khokhar ${ }^{a}$ \\ ${ }^{a}$ Faculty of Animal Husbandry and Veterinary Sciences, Sindh Agriculture University, Tandojam, Pakistan \\ ${ }^{b}$ Gomal College of Veterinary Sciences, Gomal University, D.I. Khan, Pakistan \\ (received January 31, 2012; revised April 4, 2012; accepted June 21, 2012)
}

\begin{abstract}
In order to determine the zoonotic importance of cystic echinococcosis (CE) problem in the humans, a study was conducted of patients record available at the Department of Histopathology, Liaquat University of Medical and Health Sciences, Jamshoro. Data was recorded from 1998 to 2008 by using pre-designed proforma. During that period 44 cases of hydatid cysts were recorded out of 43656 registered cases. Highest prevalence of hydatid cysts was recorded in $1999(0.21 \%)$ and the lowest in $2002(0.02 \%)$, whereas no case was registered in $2003(0 \%)$, respectively. Cystic echinococcosis was more efficiently recorded in females $(68.18 \%)$ as compared to males $(31.81 \%)$. To calculate the age wise CE in the population; the total population was divided into four age groups A (5-20 years), B (21-40 years), C (4160 years) and $\mathbf{D}$ (61 years - onward). The occurrence of CE was highest recorded in age group B (50\%) and lowest in-group D (4.54\%).
\end{abstract}

Keywords: cystic echinococcossis, Zoonotic disease, dogs

\section{Introduction}

The cestode Echinococcus is the causative agent of cystic echinococcosis (CE), or hydatidosis, which is recognized as one of the major zoonotic diseases, affecting both humans and domestic animals in various parts of the world (Gottstein and Hemphill, 1997). Hydatid disease, hydatidosis and cystic echinococcosis are all terms describing infection with the metacestode of the tapeworm, Echinococcus (Wen et al., 1993). CE occurs commonly in two forms which differ in pathology, morphology, clinical manifestations and epidemiology. One is cystic hydatid disease (CHD), caused by infection with the larval stage of Echinococcus granulosus. E. granulosus has transmitted primarily in domestic cycles between dogs and livestock, especially sheep (Wen et al., 1992). The other form is alveolar hydatid disease (AHD), caused by Echinococcus multilocularis. The most common life cycle of $E$. multilocularis involves transmission between foxes (host to adult worm) and microtine rodents as intermediate hosts (larval worm). A rare case of mixed cystic and alveolar echinococcosis (AE) was reported from China (Wen et al., 1992). Cystic echinococcosis is a zoonotic problem caused by E. granulosus, which affects larger population of humans and animals, it is the common problem of South America, Australia, the Middle East, and Mediterranean countries especially in poorly developed countries (Basgul et al., 2002). Hydatid disease is commonly found in sheep *Author for correspondence; E-mail: shoaibbaloch2002@yahoo.com and cattle farming areas of the world (Sahin et al., 2005). It can occur in any part of the body like brain, muscle tissue, kidney, bone, heart, pancreas peritoneal cavity, retro-peritoneum, soft tissue and breast but most common sites of the cyst are liver and lungs (Basgul et al., 2002). Hydatid cyst growth is slow, maturity being reached in 6-12 months (Urquhart et al., 2003). Dog is its definitive host and sheep, pigs, cattle, goats, horses, camels, wallabies, kangaroos and humans are their intermediate hosts. The humans are infected by consuming contaminated animal products (contain eggs of worm) or a person who comes in close contact with the infected dog.

The E. granulosus is one of the smallest cestodes mature after 6 weeks. Entire length of E. granulosus is only $6 \mathrm{~mm}$. After every two weeks, each mature worm sheds their last segment of the body, which contains immature eggs. The eggs are expelled out in faeces that contaminate the vegetable garden and water. Their eggs are highly resistant to weather conditions and can remain viable for about 2 years on ground (Urquhart et al., 2003). The eggs hatch in the animal's gastro intestinal tract called oncospheres, and these penetrate in intestinal wall, enter into bloodstream and from blood to vital organs of the body, where they develop watery 'blisters' called hydatid cysts. These cysts contain up to millions of tapeworm heads. Hydatid disease is non-contagious and is not spread by direct contact. 


\section{Materials and Methods}

The present study was conducted at Liaquat University of Medical and Health Sciences, Jamshoro-Sindh, Pakistan, where patients come from Hyderabad and nearby districts. The present study was conducted to check the zoonotic spread of hydatid cysts from animals to human populations and it was based on the data collection from the Department of Histopathology. It was further analyzed to check out the overall prevalence of sex wise, age wise and organ specificity.

\section{Results and Discussion}

In order to determine the prevalence of hydatid cyst problem in the humans eleven-year record (1998 to 2008) of the Department of Histopathology was checked. The data was collected according to pre-designed Proforma. Total 44 cases of hydatid cysts were found out of 43656 registered patients.

Total number of hydatid cysts recorded per year. To find out the zoonotic spread of hydatid cysts per year in the population, the total number of hydatid cyst cases within a year was recorded. The prevalence of hydatid cysts was found higher in the year 1999 followed by 2000 whereas no case was recorded in 2003 , respectively (Table 1).

Sexwise prevalence of hydatid cysts recorded per year. Total number of hydatid cysts cases were recorded in a year to find out the sex wise prevalence of hydatid cysts per year in the population. The prevalence of hydatid cysts were recorded more in females $(68.18 \%)$ as compared to males $(31.81 \%)$ (Table 2$)$.

Age wise prevalence of hydatid cysts in both sexes. To calculate the age wise prevalence of $\mathrm{CE}$ in the population, total population was divided into four age groups A (5-20 years), B (21-40 years), C (41-60 years) and $\mathbf{D}$ (61 years-onward) as shown in Table 3. Most of the cases of CE were recorded in age group B (50\%)

Table 1. Year wise prevalence of hydatid cysts recorded

\begin{tabular}{llll}
\hline \hline Year & No. of cases & Year & No. of cases \\
\hline 1998 & 5 & 2005 & 4 \\
1999 & 7 & 2006 & 3 \\
2000 & 7 & 2007 & 8 \\
2001 & 2 & 2008 & 4 \\
2002 & 1 & & \\
2003 & 0 & & \\
2004 & 3 & & \\
\hline \hline
\end{tabular}

Table 2. Prevalence of hydatid cysts recorded in both sexes

\begin{tabular}{lll}
\hline \hline Sex & No. of cases & $(\%)$ \\
\hline Male & 14 & $31.81 \%$ \\
Female & 30 & $68.18 \%$ \\
Total & 44 & - \\
\hline
\end{tabular}

Table 3. Prevalence of CE in different age groups

\begin{tabular}{lll}
\hline \hline Age group & No. of cases & $\mathbf{( \% )}$ \\
\hline A (5-20 years) & 14 & 31.81 \\
B (21-40 years) & 22 & 50 \\
C (41-60 years) & 06 & 13.63 \\
D (61 years - onwards) & 02 & 4.54 \\
Total & 44 & 100 \\
\hline \hline
\end{tabular}

followed by age group A (31.81\%), C (13.63\%) and D (4.54\%).

Prevalence of hydatid cysts in different organs. To find out the organ wise prevalence of hydatid cysts per year in the population, total recorded cases of hydatid cysts were divided according to organ specificity per year. Most of the cases of hydatid cysts were found in liver $(65.90 \%)$ followed by abdomen $(6.81 \%)$, lung (4.54\%), uterus (2.27\%), thigh (2.27\%), ovary ( $2.27 \%)$, gallbladder $(2.27 \%)$, forearm $(2.27 \%)$, endometrium $(2.27 \%)$, thyroid $(2.27 \%)$, thorax $(2.27 \%)$, spleen (2.27\%), (excisional, 2.27\%), respec-tively (Table 4).

In this study it was observed that the females were more prone to this infection (68.18\%) whereas males were less infected (31.81\%), similarly reported by Nihal and Cigde (2007). They reported that in some endemic countries, females are affected more than males because their lives bring them into contact with the parasite. The pathogenesis of parasites in female is obscure. Enrico Brunetti (2008), also reported the similar findings.

It was also noted that the age group between 20-40 years old was found to be more infected (50\%) with the hydatid cysts as compared to other age groups i.e. 520 year (31.81\%), 41-60 years $(13.63 \%)$ and 61 years to onward (5.54\%), respectively. Enrico Brunetti (2008) who reported that the individuals of all ages were infected also reported similar results. In some endemic countries, the age group of 10-35 years having higher infection may be due to their contact and playing with dogs. Berker et al. (2009) and Kalinova et al. (2005) 
Table 4. Prevalence of hydatid cysts in different organs

\begin{tabular}{lll}
\hline \hline Organ & No. of cases & $(\%)$ \\
\hline Liver & 29 & 65.90 \\
Lung & 02 & 4.54 \\
Abdomen & 03 & 06.81 \\
Uterus & 01 & 2.27 \\
Thigh & 01 & 2.27 \\
Ovary & 01 & 2.27 \\
Gallbladder & 01 & 2.27 \\
Forearm & 01 & 2.27 \\
Endometrium & 01 & 2.27 \\
Thyroid & 01 & 2.27 \\
Thorax & 01 & 2.27 \\
Spleen & 01 & 2.27 \\
Excisional & 01 & 2.27 \\
Total & 44 & 100 \\
\hline \hline
\end{tabular}

reported that the age group between $15-40$ year old was found to be more often infected with echinococcossis.

\section{Recommendations}

Poor sanitary conditions might be one of the reasons for the spread of this disease, therefore it should be suggested to ensure clean and hygienic environment. Dog is the definitive host of E. granulosus and releases the eggs of this parasite through feaces. It should be strongly suggested to avoid close contact with the dogs especially stray dogs.

The data of ten years (1998-2009) was collected from the record of the Liaquat University of Medical and Health Sciences, Jamshoro and will be further analyzed by working with simple percentages, Means, standard deviation (Wang and Peng, 2008).

\section{Conclusion}

In this study, it is concluded that $\mathrm{CE}$ occur in any part of the body but more commonly in liver, abdomen and lungs, specially females are more infected than the males, and age group between 20-40 years old are most susceptible to hydatid cysts problem.

\section{References}

Basgul, A., Kavak, Z.N., Gokaslan, H., Kullu, S. 2002. Hydatid cyst of the uterus. Infectious Diseases, Obstetrics and Gynecology, 10: 67-70.

Enrico Brunetti, M.D. 2008. Echinococcosis Hydatid Cyst. e-medicine (online article) medscape.com, 66: 262-267.

Gadea, G., Ayala, M.T., Diago, A., Cuñat, J., Garcia, de Lomas. 2000. Immunological Diagnosis of human hydatid cyst relapse: utility of the enzymelinked immunoelectrotransfer blot and discriminant Analysis. Clinical and Diagnostic Laboratory Immunology, 7: 549-552.

Gottstein, B., Hemphill, A. 1997. Immunopathology of Echinococcus. Chemical Immunology, pp. 177-208.

Kalinova, K., Proichev, V., Stefanova, P., Tokmakova, K., Poriazova, E. 2005. Hydatid bone disease: a case report and review of the literature. Journal of Orthopaedic Surgery, 13: 323-325.

Nihal, K., Arican, C. 2007. A hydatid cyst found in an uncommon site coincidentally. Pakistan Journal of Medical Sciences, (Part-I), 23: 774-776.

Sahin, E., Nayki, U., Sadik, S. 2005. Abdominal and pelvic hydatid disease during pregnancy. Archives of Gynecology and Obstetrics, 273: 58-59.

Urquhart, G.M., Armour, J., Duncan, J.L., Dunn, A.M., Jennings, F.W. 2003. Veterinary Parasitology. pp. 127-129, $2^{\text {nd }}$ edition, Blackwell Sciences Ltd., UK.

Wang, J.K., Peng, K.M. 2008. Development morphology of the small intestine of chicks. Poultry Science, 87: 2629-2635.

Wen, H., New, R.R.C., Craig, P.S. 1993. Diagnosis and treatment of human hydatidosis. The British Journal of Clinical Pharmacy, 35: 565-574.

Wen, H., Tian, W.L., Zou, P.F., Luan, M.X. 1992. A rare case of mixed cystic and alveolar hydatidosis. Transaction of the Royal Society of Tropical Medicine and Hygiene, 86: 290-291. 